\title{
BMJ Open Observational study investigating Tolerance Of Anticancer Systemic Therapy In the Elderly (TOASTIE): a protocol
}

\author{
Helen Dearden, ${ }^{1}$ Mark A Baxter (D) , ${ }^{2,3}$ Sally Martin, ${ }^{1}$ Michael Rowe, ${ }^{4}$ \\ Kieran Zucker (D) , ${ }^{1}$ Christopher Mark Jones (D) , ${ }^{1}$ Anna C Olsson-Brown, ${ }^{5}$ \\ R D Petty, ${ }^{2,3}$ Daniel Swinson, ${ }^{1}$ on behalf of NOTCH
}

To cite: Dearden H, Baxter MA, Martin S, et al. Observational study investigating Tolerance of Anticancer Systemic Therapy In the Elderly (TOASTIE): a protocol. BMJ Open 2021;11:e051104. doi:10.1136/ bmjopen-2021-051104

- Prepublication history and additional supplemental material for this paper are available online. To view these files, please visit the journal online (http://dx.doi.org/10.1136/ bmjopen-2021-051104)

Received 09 March 2021 Accepted 23 August 2021
Check for updates

(C) Author(s) (or their employer(s)) 2021. Re-use permitted under CC BY-NC. No commercial re-use. See rights and permissions. Published by BMJ.

For numbered affiliations see end of article.

Correspondence to Dr Mark A Baxter; m.z.baxter@dundee.ac.uk

\section{ABSTRACT}

Introduction The number of older adults diagnosed with cancer is increasing. Older adults are more likely to have pre-existing frailty, which is associated with greater chemotherapy-related toxicity. Early identification of those at risk of toxicity is important to reduce patient morbidity and mortality. Current chemotherapy toxicity prediction tools including the Cancer and Ageing Research Group (CARG) tool exist but are not in routine clinical use and have not been prospectively validated in a UK population. This study is the first prospective study to investigate the CARG tool in a UK population with cancer.

Methods and analysis Tolerance Of Anticancer Systemic Therapy In the Elderly is a prospective observational study of patients, aged $\geq 65$ years, commencing first-line (any indication) chemotherapy for a solid-organ malignancy. Patients receiving other systemic anticancer agents or radiotherapy will be excluded. The primary objective will be to validate the ability of the CARG score to predict grade $3+$ toxicity in this population. Secondary objectives include describing the feasibility of screening for frailty, as well as the prevalance of frailty in this population and assessing patient and clinician perception of chemotherapy toxicity risk. 500 patients will be recruited over a two year period. Baseline assessments will be recorded. At the end of the 6-month follow-up period, toxicity data will be retrospectively collected. A descriptive analysis of the recruited population will be performed. The validity of the CARG model will be analysed using receiver-operating characteristic curves and calculation of the area under the curve (c-statistic).

Ethics and dissemination The study has received ethical approval from the East of Scotland Research Ethics Service 20/ES/0114. Results will be reported in peerreviewed scientific journals and disseminated to patient organisations and media.

\section{INTRODUCTION}

The number of adults of age $>65$ years diagnosed with cancer is increasing rapidly and is expected to treble by 2040 in the UK. ${ }^{1}$

Randomised controlled trials poorly represent the general oncology population, with
Strengths and limitations of this study

- This is the first multicentre study to prospectively validate the Cancer and Ageing Research Group (CARG) score in a UK real-world population with cancer.

- The study will evaluate the feasibility of implementation of frailty screening tools within a National Health Service oncology clinic environment.

- Statistical analysis within the study will be based on a previously validated scoring system.

- The study does not account for patients receiving combination modality treatment or immunotherapy.

- The study does not explore whether interventions based on the CARG score result in improved patient outcomes.

only $20 \%$ of patients being eligible to participate and fewer than 3\% participating in clinical trials. ${ }^{2}$ Certain groups are commonly under-represented, including older patients and those with multiple pre-existing health conditions, and thus frailty. ${ }^{3}$ This results in large disparities between the data collected in clinical trials and that seen in the realworld clinical environment. ${ }^{4}$ The provision of clear and accurate information relating to both potential benefits and potentials harms of treatment is an essential factor in assisting both clinicians and patients to make informed evidence-based decisions.

Frailty in cancer has been extensively described in the literature and has been found to correlate with an increased risk chemotherapy intolerance and death. ${ }^{5}$ Although considered as distinct entities, the prevalence of frailty increases with age.

The decision to offer chemotherapy is made by the treating physician following an assessment of patient suitability. Clinicians commonly use the Eastern Cooperative 
Oncology Group performance status as a crude assessment tool for this purpose in patients of all age groups, despite it being shown to poorly correlate with frailty. ${ }^{6}$

Research into the utility of frailty scores in aiding chemotherapy decision-making is limited. Therefore, most clinical decisions are underpinned by heuristic learning obtained through clinical practice despite clinicians having a tendency to overestimate benefit and underestimate harm. ${ }^{7}$ Studies have highlighted that oncologists' predictions of grade 3 chemotherapy toxicity poorly correlated with actual treatment toxicity. ${ }^{89}$ Both underestimation and overestimation of risk of significant toxicity from chemotherapy may lead to changes in patient decisions, highlighting the need to provide accurate information. ${ }^{10}$

Failure to take account of patient frailty may result in inappropriate selection of patients for chemotherapy, a neglect to make appropriate alterations to treatment, such as dose reduction, and a lack of patient-centred intervention, culminating in adverse patient experience. ${ }^{11}$ Incorporation of frailty screening within oncology has been under review for some time and several different tools have been appraised to help predict mortality and chemotherapy toxicity, ${ }^{12}$ including the International Society of Geriatric Oncology Geriatric Screening Tool (G8) ${ }^{13}$ and Rockwood Clinical Frailty Score (CFS) ${ }^{6}$ Although these tools have all been demonstrated to have utility in identifying frailty, none have been studied as predictive markers of chemotherapy toxicity.

Two chemotherapy prediction tools that have been studied are the Cancer and Ageing Research Group (CARG) score and the Chemotherapy Risk Assessment Scale for High-Age Patients (CRASH) score. ${ }^{14}{ }^{15}$ The CRASH score takes 20-30 min to complete and due to time constraints it may not be practically feasible to incorporate this tool into non-trial National Health Service (NHS) clinical practice.

The CARG score can be used to predict grade 3-5 chemotherapy-related toxicity in patients $>65$ years of age undergoing treatment of solid-organ cancers and lymphomas ${ }^{23}$. It considers 11 domains and takes approximately $5 \mathrm{~min}$ to complete. The results of the assessment can be used to assign the risk of developing grade 3-5 chemotherapy toxicity which is expressed as both a percentage and low, intermediate or high risks. ${ }^{15}$ This information can subsequently be used in providing more personalised information related to treatment risks, which can better inform discussions and decision-making between the clinical team and the patient. ${ }^{11}$

The score was initially developed in the USA and has subsequently been adapted to include tumour specific factors relating to breast cancer. ${ }^{16}$ Subsequent research at centres in Australia questioned its ability to predict risk and it has thus far failed to enter routine clinical practice there. ${ }^{9}$ The variation in the tools utility by geographical area may be due to differences in thresholds of patient selection for chemotherapy and variation in treatments delivered, coupled to the availability of support networks.
To date, the CARG score has not been evaluated prospectively in a UK population.

In summary, we know that frailty is associated with chemotherapy intolerance and while many scoring systems exist to evaluate frailty, in practice it is rare that formal scoring assessments are utilised. Physician estimates of chemotherapy tolerance are poorly correlated to objective risk and clinical outcomes, further supporting the case for developing a validated scoring system.

This study aims to address the gap in much needed information regarding frailty and chemotherapy tolerance in the older cancer population within the UK. To our knowledge, no frailty scoring system predicting for chemotherapy toxicity has been evaluated for feasibility and validity in the UK population.

The Tolerance Of Anticancer Systemic Therapy In the Elderly study aims to validate the CARG scoring system in a UK real-world older aged population across a range of solid-organ tumour groups. Through the information we collect, we will also be able to assess the utility of other commonly used geriatric assessment tools in predicting risk of severe chemotherapy toxicity, namely the G8 and Rockwood CFS.

The CARG score was specifically chosen owing to the fact it has already been validated in the USA, and in view of its simplicity and length, it is more likely to be able to fit in to a time-constrained new patient NHS consultation. If the CARG score is validated as a tool to predict highgrade chemotherapy-induced toxicity in our UK cohort and can easily be integrated into routine clinical practice there will be significant implications for both patients and cancer services. Decisions on starting chemotherapy will be better informed by a more accurate risk assessment of toxicity. This will allow more refined dose and regimen choices ultimately leading to fewer high-grade toxicities, benefting patients' quality of life and reducing the strain on health services that would otherwise have had to manage these toxicities.

If we are unable to validate the CARG score, we will look at the utility of G8 and CFS in predicting chemotherapy toxicity in our study population.

We will also describe the prevalence of frailty in patients aged $\geq 65$ years who are commencing first-line chemotherapy in our population and demonstrate the feasibility of implementing frailty assessment in routine practice. This will help inform future NHS service development.

Finally, this study will assess the value of risk prediction tools compared with clinicians' estimates of toxicity risk, as well as exploring patients' perception of risk associated with chemotherapy in this population.

\section{METHODS AND ANALYSIS}

This is a UK multicentre prospective observational study (non-randomised) which will recruit patients aged $\geq 65$ years who are commencing first-line neoadjuvant, adjuvant or palliative chemotherapy for a solid-organ malignancy with a prognosis of $>3$ months. 
Box 1 Inclusion and exclusion criteria for the Tolerance Of Anticancer Systemic Therapy In the Elderly study

\section{Inclusion criteria}

- Age $\geq 65$ years.

- About to start first-line chemotherapy for a solid-organ malignancy.

- Chemotherapy given for any intent, for example, neoadjuvant, adjuvant or palliative.

- Clinician estimated survival of $>3$ months.

- Able to comprehend and complete questionnaire.

\section{Exclusion criteria}

- Unable to provide informed consent.

- Participant is enrolled in another clinical trial.

- Prior chemotherapy for any indication.

- Receiving concurrent radiotherapy or immunotherapy. Patient who have completed radiotherapy prechemotherapy are eligible.

The study plans to recruit 500 patients over the course of 24 months using centres which are part of the National Oncology Trainee Collaborative for Healthcare research (NOTCH) network. ${ }^{17}$ A prestudy survey sent to 21 centres indicated this number is feasible to recruit. Further details on sample size calculations can be found in the Statistical Analysis Plan below. Participants will be followed up over a period of 6 months or until completion of treatmentwhichever is earliest. Recruitment will commence in April 2021 and is anticipated to complete in April 2022.

The CARG score has not been validated in a UK population therefore the study does not alter treatment decision. Participants will not be required to undergo any additional investigations or treatments. The local clinical team responsible for the patient will be able to access and use the additional information gathered, and address any issues as needed.

\section{Study population}

Participants will be $\geq 65$ years old commencing first-line chemotherapy with any treatment intent for a solid-organ malignancy. The inclusion and exclusion criteria are shown in box 1 .

\section{Recruitment process}

Potential study participants will be identified by the care team in each participating site. Each participating site will develop their own strategies for identifying participants. Participant advertising material can be distributed for local promotion. Potential participants will be provided with a patient information sheet (PIS) to read prior to their clinic review where possible or given time to read this literature after the clinic in all other cases (online supplemental file 1).

\section{Consent and registration}

Consent to participate in the study will take place during a standard clinic appointment. During the new patient clinic review, an authorised member of the research team will discuss participation in this study and seek consent once the participant has had sufficient time to consider the PIS and ask any questions. Questions regarding the study will be answered to the satisfaction of the potential participant in a language understandable to them. As this is an observational study only, it is deemed reasonable to discuss the study and consent during a single clinic visit if that fits the pathway of the centre. In the current climate, returning for additional study visits also puts participants at greater risks given that hospitals are known to act as a reservoir for SARS-CoV-2, the causative agent for COVID19. In some centres where patients are consented for chemotherapy at a second appointment, the participant could consent to the study at this visit.

Assenting participants will be asked to document their consent by initialling the boxes on the consent form, printing and signing their name, and dating the form. The investigator must complete the header details on the consent form and check that the participant has completed the consent form correctly. The investigator will also print and sign their name and date the form contemporaneously.

The participant will be assigned a study number. All study IDs will be of the format XXXYYY where XXX is the centre code and YYY is the participant number.

The original consent form will be filed in the investigator site file. A copy of the consent form will be given to the participant and a copy will be retained in the participant's notes.

The principal investigator (PI) retains overall responsibility for the informed consent of participants at their site and must ensure that any person delegated responsibility to participate in the informed consent process is duly authorised, trained and competent to do so according to the ethically approved protocol, principles of good clinical practice (GCP) and Declaration of Helsinki 1996.

The right of a participant to refuse participation without giving reasons must be respected. The participant must remain free to withdraw at any time from the study without giving reasons and without prejudicing his/her further treatment. If after consenting to participate, the patient opts not to have treatment or is deemed not suitable for treatment, this will be recorded and no further data will be collected. At no point during the process of obtaining informed consent must the researcher or any member of the research team coerce or unduly influence the participant to take part in the study. None of the oral or written information concerning the study should contain any language that causes the participant to waive or appear to waive any legal rights, or that releases or appears to release the investigator, the institution, the sponsor or their agents from liability for negligence.

The PIS and consent form given to the participant must be the most current versions approved by the Research Ethics Committee. The version number and date must be printed on the document.

\section{Study assessments}

Data will be collected by the direct care team via structured forms which will be completed during the delivery 


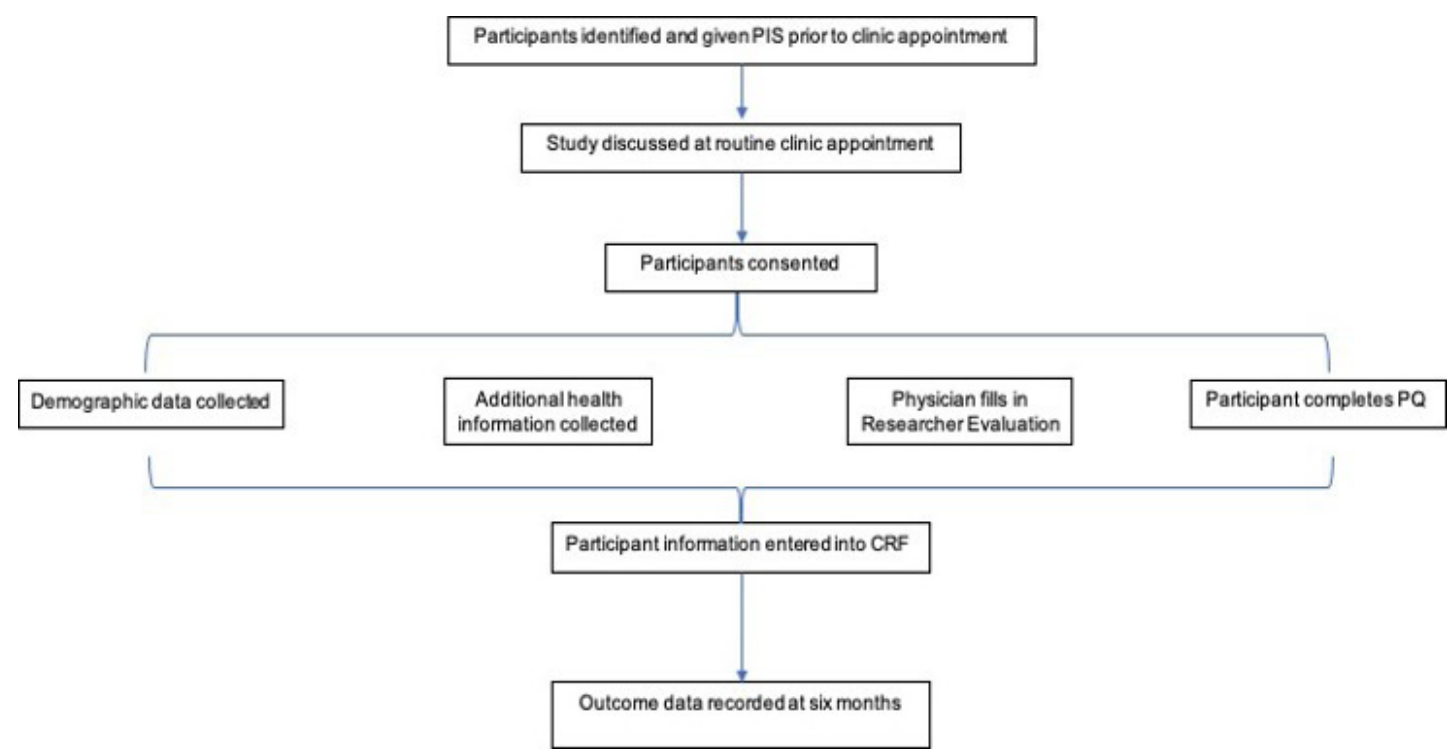

Figure 1 TOASTIE study flowchart. CRF, case report form; PIS, patient information sheet; PQ, patient questionnaire; TOASTE, Tolerance Of Anticancer Systemic Therapy In the Elderly.

of routine care to the participants enrolled within the study. The study flowchart is shown in figure 1. The CARG score will be calculated using the established predefined scores and standardised toxicity severity definitions (Common Terminology Criteria for Adverse Events (CTCAE) V.5). This will limit variation in intersite and interuser assessment.

Documents will be locally collected at each of the study sites and manually inputted into the relevant case report form (CRF). The input information will remove all patient identifiable information and will be stored against a unique study specific ID. On completion of the data collection at each site, the data will be securely transferred to the analysis environment where the data will be collated prior to analysis.

\section{Study pathway}

During a standard clinic appointment, following consent for chemotherapy, each participant will be asked additional health questions (AHQ) (online supplemental file 2). This is estimated to take $<10 \mathrm{~min}$. This information is of additional clinical value and available to the treating physician. Information collected in the AHQ should be recorded in the BASELINE AHQ CRF. Information should be collected prior to commencing cycle 1 of chemotherapy.

After completion of the clinic visit the participant will be provided with a participant questionnaire (PQ) (online supplemental file 3) to complete. This is estimated to take $<10$ minutes to complete. This will be returned to an authorised member of the research team. Information collected from the PQ should be recorded in the BASELINE PQ CRF. Information should be collected prior to commencing cycle 1 of chemotherapy. The CARG score will be calculated retrospectively from the baseline data at the end of the study period.
The authorised member of the research team collecting the information in the AHQ will complete BASELINE RE CRF (online supplemental file 4). This information should be collected following the end of the clinic consultation, and prior to receiving the results of the PQ.

The results of routine assessments and investigations performed prior to cycle 1 of chemotherapy should be recorded in the BASELINE DATA CRF once consent has been obtained. This may be after treatment has commenced. Where an investigation listed on the CRF has not been performed as part of routine care this should be documented, but an investigation should not be ordered for the purpose of this study if it has not been performed during routine care.

Participants will be assessed during treatment as per local standard care. Toxicity will be assessed according to CTCAE V.5 and only grade 3 or above toxicities will be recorded in the progress CRF. Details of hospital admissions will be obtained from the medical records retrospectively.

The PROGRESS CRF should be completed by the time of chemotherapy completion or by 6 months-whichever is earliest.

\section{Outcome measures}

Primary outcome

1. To validate the CARG score's ability to predict grade $3-5$ toxicity in patients aged $\geq 65$ years who are receiving first-line chemotherapy in the UK national health system.

\section{Secondary outcomes}

1. Describe the prevalence of frailty in patients of age $\geq 65$ years commencing first-line chemotherapy in the UK. 
2. Assess the utility of the G8 frailty assessment and Rockwood frailty score in predicting the occurrence of grade $3-5$ toxicity.

3. Demonstrate the feasibility of implementing frailty assessment in routine practice.

4. Describe patient and clinician perception of toxicity risk associated with chemotherapy.

\section{Statistical analysis plan}

As the study is an observational study, the sample size calculation has been made based on precision as opposed to power as is typical for interventional studies. This attempts to estimate the population size needed to gain a specified level of precision of the CIs. This calculation has been made using the $\mathrm{R}$ Shiny web application 'precisely'. ${ }^{18}$ Calculations used risk rates derived from the original 500 patient development cohort of the CARG study. ${ }^{15}$ The lowest pairwise difference in risk for grade 3 or more toxicity was between the low CARG $(30.47 \%)$ and intermediate CARG $(51.54 \%)$ groups with a risk difference of $21.07 \%$. The same study also identified $50 \%$ of patients as being in the intermediaterisk group and $25 \%$ each in the other two groups. Based on a 500 patient cohort, this would yield on average 125 low-risk and 250 intermediate-risk groups. Based on these numbers, precision of the $95 \%$ CI would be estimated at 0.2 . Even assuming a $15 \%$ dropout rate of participants this would give a precision of 0.23 . Both of these would be sufficient to identify the expected scale of risk difference and the majority of clinically relevant risk differences.

Prior to commencing analysis, a detailed statistical analysis plan will be produced. The CARG score will be calculated from 11 prechemotherapy variables. ${ }^{15}$ Patients will be categorised into low (0-5), intermediate (6-9) and high (10-19) risk. Observed grade 3-5 toxicity rates between groups will be compared using a $\chi^{2}$ test of proportions. We will then assess the validity of the model by composing receiver-operating characteristic curves and calculating the area under the curve (c-statistic) for the CARG score.

A descriptive analysis of the prevalence of frailty in the population and the feasibility of implementing frailty assessment will be performed. Patient and clinician perception of risk will be compared using a paired t-test of the baseline perceived percentage risk.

Data missing from the CRF will, where possible, be completed retrospectively from written records.

\section{Patient and public involvement}

The PIS, consent form and patient questionnaire have been reviewed by older adult patients ( $\geq 65$ years as per inclusion criteria of study) who are either receiving systemic anticancer treatment or have previously received treatment (for any tumour type and with all intents, for example, neoadjuvant, adjuvant and palliative).
ETHICS AND DISSEMINATION

\section{Ethical approval}

This TOASTIE study has been approved by the East of Scotland Research Ethics Service 20/ES/0114 with protocol V.1.2 dated 16 November 2020.

The study will be conducted in accordance with the principles of GCP as applicable under UK regulations, the NHS Research Governance Framework and through adherence to study standard operating procedures. All members of the research team will be GCP-trained. The study will also be performed in accordance with the recommendations guiding physicians in biomedical research involving human participants adopted by the 18th World Medical Assembly, Helsinki, Finland, 1964, amended at the 52nd World Medical Association General Assembly, Edinburgh, UK, October 2000.

Patients will be pseudoanonymised. The care team will be responsible for assigning study numbers to consented participants as per the protocol. No patient identifiable data will be transferred to the research team. Paper questionnaires completed by participants will be kept securely in a locked drawer in a locked security-controlled office, in the investigator site file at each recruiting site by the care team.

Electronic data will be stored at each site in a designated password-protected folder, on secure passwordprotected NHS servers. Only relevant study personnel on the delegation log will have access to this.

Linked-anonymised data will be shared via nhs.net (which is a secure mail system regularly used by clinicians to share patient data) to a secure mailbox to which only one person has access. It will be transferred to a secure, password-protected study-specific domain on a high-secure tertiary centre NHS Trust (The Leeds Teaching Hospitals NHS Trust) server, to which only relevant study personnel will have access. The master Microsoft Excel document within this domain will additionally remain password-protected.

\section{Data storage and security}

All information collected during the course of the study will be kept strictly confidential. Information will be held securely on paper and electronically. The research team will comply with all aspects of the 2018 Data Protection Act.

Investigator site files, including copies of CRFs and eligibility/link-anonymisation forms, will be stored securely at NHS sites by each PI for a period of 10 years. This may be either in physical or electronic form as per local NHS policy. The Leeds Teaching Hospitals NHS Trust will store link-anonymised 'Confirmation of Consent' forms and CRFs for 10 years. After 10 years, arrangements for confidential destruction of data and documents that are due to be disposed of will be made by the sponsor.

This study is conducted within the framework of information governance (IG) good practice.

The data analysis will be undertaken in two settings: either within the NHS by employees of the organisation or university researchers within NHS-approved secure data environments. All staff will have undergone appropriate training on IG and will be given access to the research data files for the 
purposes of this study. Research data files will be stored on password-protected NHS computers on secure networks or other NHS-approved secure cloud infrastructure.

University colleagues may have access to increased resources for delivering this work, in particular human capital that will allow the fastest route to realising the potential patient benefit of this research data. This process will follow the formal data release process in place at Leeds Teaching Hospital Trust.

\section{Statement of indemnity}

This study is sponsored by the Leeds Teaching Hospital Trust who will be liable for negligent harm caused by the design of the study. The NHS has a duty of care to participants, whether or not the participant is taking part in a study, and the NHS remains liable for clinical negligence and other negligent harm to participants under this duty of care.

\section{Dissemination of results}

Following completion of follow-up and analysis of data, results will be communicated in academic forums such as presentation at academic meetings and publication in journals. Results will also be communicated to the public through patient networks. Publication will be under joint authorship on behalf of NOTCH. We used the Standard Protocol Items: Recommendation for Interventional Trials (SPIRIT) checklist when writing this report. ${ }^{20}$

\section{Author affiliations}

'Leeds Cancer Centre, St James's University Hospital, Leeds Teaching Hospitals NHS Trust, Leeds, UK

${ }^{2}$ Division of Molecular and Clinical Medicine, University of Dundee School of Medicine, Dundee, UK

${ }^{3}$ Tayside Cancer Centre, Ninewells Hospital, NHS Tayside, Dundee, UK

${ }^{4}$ Plymouth Oncology Centre, Derriford Hospital Cancer Services Department, Plymouth, UK

${ }^{5}$ Clatterbridge Cancer Centre NHS Foundation Trust, Bebington, UK

Correction notice This article has been corrected since it was published. Steering group members added under the NOTCH authorship.

Twitter Mark A Baxter @majbaxter and Christopher Mark Jones @DrCMJones

Collaborators NOTCH Steering committee: Clair Brunner, Fiona Smith, Anna Lewis, Sarah Taylor, Lisa Rodgers, Anthea Cree, Eleanor Smith.

Contributors HD: designed the study and wrote the protocol; MAB: drafted the manuscript, assisted in the study design and writing of the protocol; KZ: designed the statistical analysis plan for the study; SM, MR, CMJ, ACO-B, RDP, DS: assisted in the study design and writing of the protocol. All authors approved the final version.

Funding MAB is a Clinical Academic Fellow funded by the Scottish Government Chief Scientist Office. CMJ is funded by a Wellcome Trust Clinical Research Fellowship.

Competing interests $A C O-B$ has received honoraria from BMS, MSD and Roche outside the scope of this work. MR has received honoraria from MSD, Servier and Astellas Pharma outside the scope of this work. RDP has undertaken speaking, consulting and advisory roles for Eli Lilly, BMS, Pfizer, Sanofi, Servier; and received research funding (not related to the work in this manuscript) from Astra Zeneca, Roche, MSD, Merck serrano, Eli Lilly, Five Prime Therapeutics, Clovis, Boston Biomedical and Janssen.

Patient consent for publication Not applicable.

Provenance and peer review Not commissioned; externally peer reviewed.

Supplemental material This content has been supplied by the author(s). It has not been vetted by BMJ Publishing Group Limited (BMJ) and may not have been peer-reviewed. Any opinions or recommendations discussed are solely those of the author(s) and are not endorsed by BMJ. BMJ disclaims all liability and responsibility arising from any reliance placed on the content. Where the content includes any translated material, BMJ does not warrant the accuracy and reliability of the translations (including but not limited to local regulations, clinical guidelines, terminology, drug names and drug dosages), and is not responsible for any error and/or omissions arising from translation and adaptation or otherwise.

Open access This is an open access article distributed in accordance with the Creative Commons Attribution Non Commercial (CC BY-NC 4.0) license, which permits others to distribute, remix, adapt, build upon this work non-commercially, and license their derivative works on different terms, provided the original work is properly cited, appropriate credit is given, any changes made indicated, and the use is non-commercial. See: http://creativecommons.org/licenses/by-nc/4.0/.

\section{ORCID iDs}

Mark A Baxter http://orcid.org/0000-0002-5773-8650

Kieran Zucker http://orcid.org/0000-0003-4385-3153

Christopher Mark Jones http://orcid.org/0000-0002-4513-4964

\section{REFERENCES}

1 Maddams J, Utley M, Møller H. Projections of cancer prevalence in the United Kingdom, 2010-2040. Br J Cancer 2012;107:1195-202.

2 Denicoff AM, McCaskill-Stevens W, Grubbs SS, et al. The National cancer Institute-American Society of clinical oncology cancer trial Accrual symposium: summary and recommendations. J Oncol Pract 2013;9:267-76

3 Townsley CA, Selby R, Siu LL. Systematic review of barriers to the recruitment of older patients with cancer onto clinical trials. J Clin Oncol 2005;23:3112-24.

4 Murthy VH, Krumholz HM, Gross CP. Participation in cancer clinical trials: race-, sex-, and age-based disparities. JAMA 2004;291:2720-6.

5 Handforth C, Clegg A, Young C, et al. The prevalence and outcomes of frailty in older cancer patients: a systematic review. Ann Oncol 2015;26:1091-101.

6 Denholm M, Corrie P, Qian W, et al. The Rockwood geriatric clinical frailty scale is a more discriminatory tool for assessing older cancer patients compared with standard oncology performance status scales. European Journal of Surgical Oncology 2017;43:2236.

7 Hoffmann TC, Del Mar C. Clinicians' expectations of the benefits and harms of treatments, screening, and tests: a systematic review. JAMA Intern Med 2017;177:407-19.

8 Alibhai S, Manokumar T, Aziz S, et al Does the hurria/CARG tool or VES-13 predict grade $3+$ toxicities in men undergoing chemotherapy for metastatic prostate cancer? J Geriatr Oncol 2013;4:S37.

9 Moth EB, Kiely BE, Stefanic N, et al. Predicting chemotherapy toxicity in older adults: comparing the predictive value of the CArG toxicity score with oncologists' estimates of toxicity based on clinical judgement. J Geriatr Oncol 2019;10:202-9.

10 Kuchuk I, Bouganim N, Beusterien K, et al. Preference weights for chemotherapy side effects from the perspective of women with breast cancer. Breast Cancer Res Treat 2013;142:101-7.

11 Hurria A, Togawa K, Mohile SG, et al. Predicting chemotherapy toxicity in older adults with cancer: a prospective multicenter study. JCO 2011;29:3457-65.

12 Harari DK Tet al. Comprehensive Geriatric Assessment and Available Tools'. In: Ring A, Kalsi T, Harari D, eds. Problem solving in older cancer patients. Oxford: Atlas Medical Publishing, 2016: 33-42.

13 Bellera CA, Rainfray M, Mathoulin-Pélissier S, et al. Screening older cancer patients: first evaluation of the G-8 geriatric screening tool. Ann Oncol 2012;23:2166-72.

14 Extermann M, Boler I, Reich RR, et al. Predicting the risk of chemotherapy toxicity in older patients: the chemotherapy risk assessment scale for High-Age patients (crash) score. Cancer 2012;118:3377-86

15 Hurria A, Togawa K, Mohile SG, et al. Predicting chemotherapy toxicity in older adults with cancer: a prospective multicenter study. $J$ Clin Oncol 2011;29:3457-65.

16 Hurria A, Magnuson A, Gross C. Abstract GS6-04: development and validation of a chemotherapy toxicity (chemo tox) risk score for older patients (PTS) with breast cancer (bc) receiving adjuvant/neoadjuvant treatment (adjuvant tx): a R01 and BCRF funded prospective multicenter study. Cancer Research 2019;79:GS6-04-GS6-04. 
17 Jones CM, Olsson-Brown A, Dobeson C. Notch: the National oncology trainees collaborative for healthcare research. Clin Oncol 2020;32:632-5.

18 The precisely tool. Available: https://github.com/malcolmbarrett/ precisely
19 Rothman KJ, Greenland S. Planning study size based on precision rather than power. Epidemiology 2018;29:599-603.

20 Chan A-W, Tetzlaff JM, Gøtzsche PC, et al. Spirit 2013 explanation and elaboration: guidance for protocols of clinical trials. $B M J$ 2013;346:e7586. 